\title{
THERMAL TRANSITIONS AND SOLIDIFICATION KINETICS OF POLY(LACTIC ACID) AND BLENDS WITH EPOXIDIZED NATURAL RUBBER
}

Leno Mascia $^{* a}$, Barry Haworth $^{a}$, Adriano Vignali $^{b}$, Rosario Megna $^{c}$, Domenico Acierno $^{c}$, Pietro Russo ${ }^{d}$

${ }^{a}$ Department of Materials, Loughborough University, Loughborough LE11 3TU, UK.

${ }^{b}$ Department of Chemical, Materials and Production Engineering, University of Naples Federico II, Piazzale Vincenzo Tecchio, 80125 Naples, Italy.

${ }^{c}$ CRdC Tecnologie Scarl, Via Nuova Agnano 11, 80125 Naples, Italy

${ }^{d}$ Institute for Polymers, Composites and Biomaterials, National Research Council, Via Campi Flegrei 34, 80078 Pozzuoli, Naples, Italy.

\begin{abstract}
The reactivity of polylactic acid (PLA) with epoxidized natural rubber (ENR50) was confirmed by monitoring the changes in melt viscosity during mixing and by TGA runs.

The plasticization resulting from a partial miscibilization of ENR50 during mixing was found to have opposite effects on the crystallization of the PLA matrix, depending on the temperature scanning mode. An increase in heat of crystallization $(\Delta H c)$ with increasing temperature scanning rate was observed when heated from the glassy state, while a corresponding reduction in $\Delta H c$ was obtained in cooling scans from the melt.

Studies on the kinetics of events have shown that the classical Kissinger plots for crystallization by heating from the glassy state display a variable activation energy for the case of the reactive blend. The vitrification kinetics, on the other hand, could be modelled quite accurately with the Mahadevan method for all systems, including an intrinsically amorphous PLA.
\end{abstract}

Keywords: Crystallization kinetics, De-vitrification, Epoxidized natural rubber, Poly(lactic acid), Vitrification.

\footnotetext{
* Corresponding author:

Leno Mascia

E-mail address: 1.mascia@lboro.ac.uk
} 


\section{Introduction}

Polymer blending has been widely explored over a number of years both in industrial applications and for academic research, particularly as a means of providing a mechanism to increase the ductility and toughness of brittle polymers. The incorporation of a rubbery component in a glassy matrix has also been exploited for the manufacture of shape memory products through the formation of a semi-interpenetrating network [1].

The blending approach for property enhancement has been extended in more recent years to biodegradable materials, especially polycaprolactone (PCL) and poly(lactic acid) (PLA). In the majority of cases the blends have been produced in binary combinations with polymers derived from petrochemicals [2-9]. Gajria et al have shown that PLA is thermodynamically miscible with poly(vinyl acetate) (PVAc) over the entire concentration range, and large increases in elongation at break were observed for concentrations greater than 20 w\% PVAc [4]. The weight loss resulting from biodegradation, however, was found to decrease drastically even at very low PVAc concentrations, i.e. 5 w\%. Particularly relevant for the present study is a report by Zhang et al. concerning the crystallization of poly(L-lactic acid) in blends with ethylene/methyl acrylate/glycidyl methacrylate terpolymer (EGMA), and the work of Oyama concerned with the toughening of polylactide by the addition of an epoxy-functionalized grafted acrylonitrile-butadiene-styrene (ABS-g-GMA) particles [2, 5]. Confirmation of the reaction taking place between the epoxy groups of the auxilary polymer component and PLA was obtained in both studies.

A review of blends of PLA, aiming particularly at toughening systems, has been published more recently by Liu and Zhang [10]. Other related studies have been focussed on systems containing only biodegradable polymers [11 - 13].

In a series of successive DSC runs from 0 to $200{ }^{\circ} \mathrm{C}$, Cao et al. have shown that the miscibility of blends of PLA with poly(hydroxyl ether ester) increases considerably through transesterification reactions taking place during the heating-cooling cycles. Such heat treatments caused the suppression of cold crystallization and the disappearance of melting peaks for the PLA component [14].

Some studies have been reported recently on the toughening of PLA and property modification of PCL in binary combinations with epoxidized natural rubber (ENR) with the view to produce biodegradable blends, taking advantage of the advanced state of oxidation of the natural rubber, which are intermediate products of the aerobic molecular breakdown [15 17]. Mishra et al. have studied the effects of adding a peroxide to blends of PCL and epoxidized natural rubber (ENR) as a means of producing cross-linked networks and have 
shown that such systems have a higher level of memory than the equivalent non-crosslinked systems [16]. At the same time an increase in level of crystallinity was found for the PCL phase, which can be ascribed to the concomitant reduction in molecular weight of the polymer. In studies of the post-gelation reactions of cross-linked ENR systems, Mascia et al. [18] have found extensive degradation resulting from the opening of the oxirane groups to produce vicinal $\mathrm{OH}$ groups, catalysed by the presence of $\mathrm{COOH}$ in neighbouring chains.

In the present work the effects of thermally induced interactions between the components of a blend of PLA with a small amount of ENR50 are examined using differential scanning calorimetry to determine any influences on the crystallization and vitrification kinetics. Previous studies on these systems have addressed primarily the mechanical properties aspects and have generally used quantities of ENR larger than $10 \mathrm{w} \%$ $[14,17]$. Other studies have been reported on the effects of plasticizers in blends with PLA, which were aimed particularly at increasing the ductility [19 - 21]. The use of plasticizers, however, causes a large reduction in the glass transition temperature ( $\mathrm{Tg}$ ) and decreases considerably the yield strength, in addition to other expected undesirable effects related to volatilization and migration phenomena. Blends containing only small amounts of a rubbery polymer would overcome these drawbacks, while the use of an epoxidized natural rubber (ENR) can be expected to mitigate the deterioration of bio-degradation characteristics identified in blends with petrochemical derived polymers [6]. However, there is only a relatively scanty knowledge on the effects cooling rate on crystallization kinetics of PLA in blends and very little is known about the vitrification and devitrification kinetics of PLA based systems in general. In the present study the authors intend to obtain new insights on the effects of relatively low concentrations of ENR on the solidification kinetics of blends with PLA, as well as to provide useful data for manufacturing processes.

\section{Experimental}

\subsection{Materials}

The polymers used in this study were respectively: a) Crystalline poly(lactic acid) (PLA), under the trade name Ingeo Biopolymer 6202D, consisting of 98 \% L-lactide units and with a weight average molecular weight $(\overline{\bar{M}} \mathrm{w})$ in the region of $140 \mathrm{kDa}$. b) Epoxidized natural rubber (ENR50), Epoxyprene 50, with a 50 mol\% nominal level of epoxidation and Mooney viscosity ML(1'14')100_C570-90, manufactured by Muang Mai Guthrie Public Limited Company of Thailand, and Natural Rubber (Standard Malaysian Rubber CV 60, were kindly 
donated by Tun Abdul Razak Research Centre. c) Amorphous grade of PLA from Natureworks LLC (Ingeo 4060D, Mw = 94 kDa, Mn = 57 kDa).

\subsection{Blend composition and mixing procedure}

A binary blend of crystalline PLA with ENR50 at 90:10 weight ratio, coded "System A" as well as PLA controls, were prepared using a 50 cc Haake mixer at $180{ }^{\circ} \mathrm{C}$ for 30 minutes. The state of the mix was monitored by measuring of torque exerted by the rotors during the mixing cycle. The PLA granules were dried at $65{ }^{\circ} \mathrm{C}$ for 24 hours for the preparation of the samples and melt mixed using a rotor speed of $80 \mathrm{rpm}$.

\subsection{Thermal analysis}

In the first instance the calorimetric characterization of the materials was performed using a DSC Q20 TA Instruments. Samples, enclosed in suitable aluminum pans, were subjected to a three-step thermal cycling protocol under controlled conditions to erase their previous thermal history. In details, samples approximately $10 \mathrm{mg}$ in weight were heated from $-80^{\circ} \mathrm{C}$ to $180^{\circ} \mathrm{C}$ at a heating and cooling rates of $10^{\circ} \mathrm{C} / \mathrm{min}$, followed by a cooling cycle to $-80^{\circ} \mathrm{C}$ after a 2 minutes isotherm at $180^{\circ} \mathrm{C}$ and by a re-heating cycle over the same temperature range.

Thermogravimetric measurements were carried out in nitrogen atmosphere using a TA instrument TGA apparatus, mod Q5000. The variation of the weight as a function of the temperature was monitored for each sample subjected to a heating rate of $20^{\circ} \mathrm{C} / \mathrm{min}$ between room temperature and $700{ }^{\circ} \mathrm{C}$.

The solidification kinetics of the materials were then analysed by performing nonisothermal experiments using both cooling and heating scans. For the heating mode experiments about $10 \mathrm{mg}$ accurately weighed samples were sealed into DSC capsules, then heated on a hot plate at $200{ }^{\circ} \mathrm{C}$ for 10 minutes and subsequently quenched in icy water for at least 10 minutes at a temperature around $2-3{ }^{\circ} \mathrm{C}$. After removing the surface water the capsules were placed in the DSC instruments and runs were made from 0 to $180{ }^{\circ} \mathrm{C}$ at different heating rates varying from 0.5 to $20^{\circ} \mathrm{C} / \mathrm{min}$. Similarly, weighed samples of the same materials and in the same amounts were placed in the DSC instrument and heated from room temperature to $180^{\circ} \mathrm{C}$ at $10{ }^{\circ} \mathrm{C} / \mathrm{min}$, followed by a 2 minutes isotherm before performing 
measurements at different cooling rates over the same range 0.5 to $20{ }^{\circ} \mathrm{C} / \mathrm{min}$. Experiments at $20^{\circ} \mathrm{C} / \mathrm{min}$ were repeated using $5 \mathrm{mg}$ samples to verify the reproducibility of data.

\section{Results and discussion}

\subsection{Materials melt-mixing characteristics}

The data recorded during the mixing studies, together with the results of TGA experiments are shown in Fig. 1.

a)

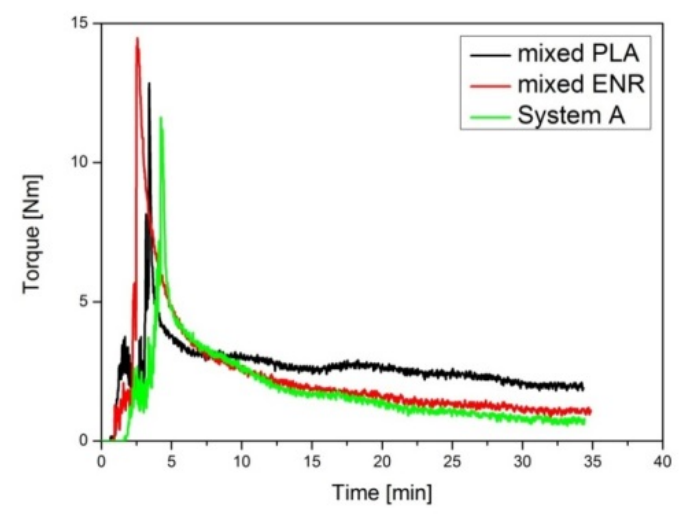

b)

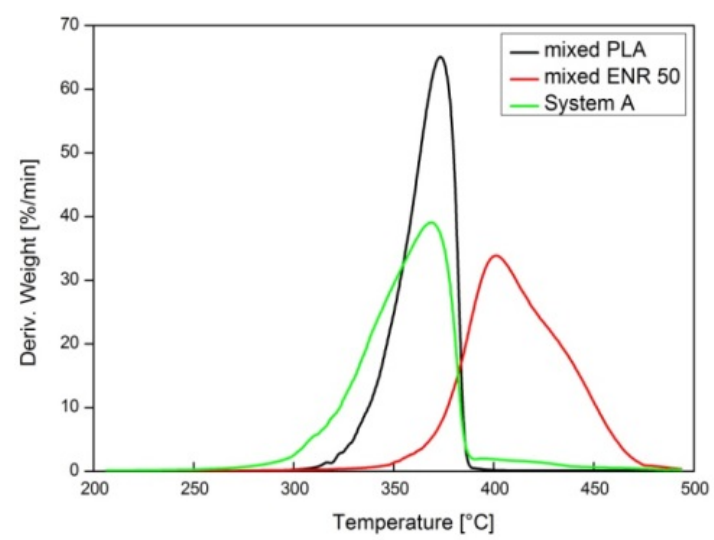

Fig. 1. a) Torque evolution during processing in the Haake mixer of System A, blend of crystalline PLA and ENR50. b) DTGA weight losses of materials after mixing.

The data in Fig. 1 (a) show that the mixing run for the PLA control display a lower decrease in torque with time than ENR 50 and an even larger reduction for System A. While the overall decrease in viscosity during processing can be attributed to typical mechanodegradation behaviour through chain scission reactions, the occurrence of intermediate shallow bumps in the torque traces of the PLA sample may be a characteristic feature of the material displaying a combination of chain scission and intramolecular transesterification reactions [22].

The larger reduction in torque exhibited by ENR50 is the result of chain scission reactions through the formation of carbonyl groups, assisted by the presence of epoxy groups in the chains as intermediate species, as identified in previous work [19]. The even lower torque values for System A relative to those exhibited by either component of the blends can be ascribed to the catalytic effects of terminal $\mathrm{COOH}$ groups in the PLA products on the chain scission degradation of both PLA and ENR 50 components. 
An indication of the effect of the reactions taking place during processing is reflected on the properties of the materials examined can be obtained from the data in Fig. 1(b). The DTGA traces for runs carried out on the processed materials show that although ENR50 is more thermally stable than PLA the thermal degradation of the blend (System A) starts at a temperature even lower. The TGA data, therefore, confirm that the lower torque values recorded for System A during mixing are to be attributed to the greater susceptibility to thermal degradation of this system, arising from ester exchange reactions of PLA with ENR50 and the catalytic effect on the degradation of both ENR50 and PLA.

It should be noted that the samples of System A were opaque and SEM examination revealed the presence of dispersed domains in the region of 4 - $10 \mu \mathrm{m}$.

\subsection{Thermal characteristics by DSC analysis}

The DSC thermograms obtained for the systems based on the crystalline PLA are shown in Fig. 2 and summarized in Table 2.

The details of the DSC thermograms and the related data indicate that the thermal history has a significant effect on events in both the glassy state and in the melting and crystallization processes. These results bring out also some peculiar features, such as the sporadic effect of the rubbery component on the cold crystallization temperature and the disappearance of the double melting peak in the second heating cycle. The erratic behaviour of PLA blends has been reported also by other authors. For instance, in the cited studies by Zhang et al. it was found that a double peak appeared in the second heating cycle except for the blends containing $20 \%$ EGA [2]. The formation of a smaller peak at a lower temperature has been widely reported for PLA systems, particularly for systems containing plasticizers [20, 21] including dissolved $\mathrm{CO}_{2}$ for foaming purpose [23], and is attributed to the segregation of less perfect crystals. 
a)

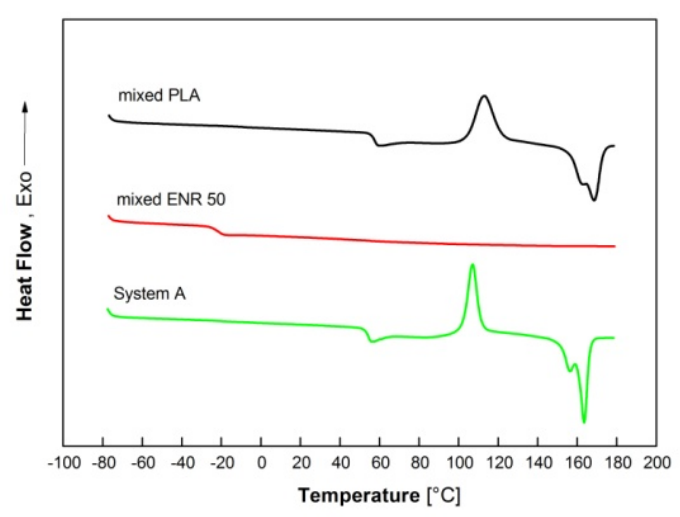

b)

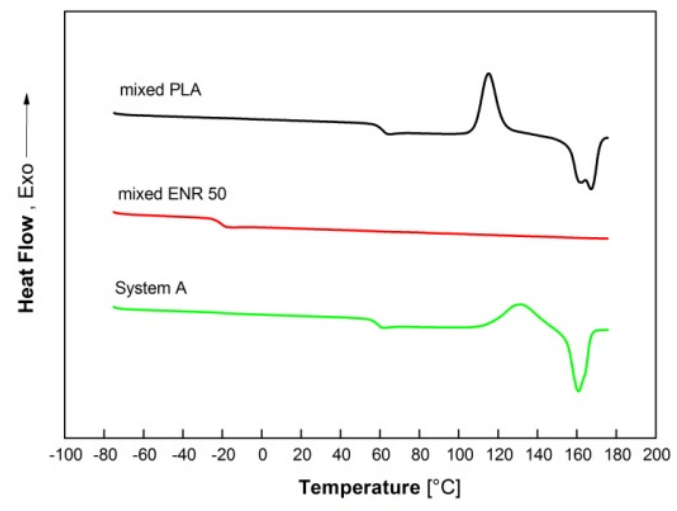

Fig. 2. DSC thermograms for crystalline PLA, ENR 50 and System A: (a) First heating and (b) second heating scan, both at $10^{\circ} \mathrm{C} / \mathrm{min}$.

\section{Table 2}

Summarized data from Fig. 2 obtained by DSC analysis of the materials based on crystalline PLA carried out within 5 days the preparation on the Haake mixer. (Note: The $\Delta H$ data for System A are the normalized enthalpy values with respect to the content of PLA).

\begin{tabular}{lcccccc}
\hline Materials & $\mathrm{T}_{\mathrm{g}}\left({ }^{\circ} \mathrm{C}\right)$ & $\mathrm{T}_{\mathrm{cc}}\left({ }^{\circ} \mathrm{C}\right)$ & $\mathrm{T}_{\mathrm{m}, 1}\left({ }^{\circ} \mathrm{C}\right)$ & $\mathrm{T}_{\mathrm{m}, 2}\left({ }^{\circ} \mathrm{C}\right)$ & $\Delta \mathrm{H}_{\mathrm{cc}}(\mathrm{J} / \mathrm{g})$ & $\Delta \mathrm{H}_{\mathrm{m}}(\mathrm{J} / \mathrm{g})$ \\
\hline mixed PLA $-1^{\text {st }}$ heating & 57.7 & 113.1 & 162.4 & 168.6 & 29.89 & 33.39 \\
mixed PLA $-2^{\text {nd }}$ heating & 61.7 & 115.2 & 164.7 & 167.2 & 30.34 & 35.36 \\
mixed ENR50 - $1^{\text {st }}$ heating & -21.4 & - & - & - & - & - \\
mixed ENR50 - $2^{\text {nd }}$ heating & -20.2 & - & - & - & - & - \\
System A $-1^{\text {st }}$ heating & 54.5 & 107.1 & 156.5 & 163.5 & 26.86 & 35.82 \\
System A $-2^{\text {nd }}$ heating & 59.3 & 132.0 & 160.9 & - & 28.00 & 31.15 \\
\hline
\end{tabular}

The absence of the double peak for System A in the second heating cycle alludes to a possible effect of further reactions between PLA and ENR 50, as can be deduced also from the DTGA traces in Fig. 1 (b). This possibility is evidenced also by the formation of a broader and delayed crystallization exotherm during the second heating cycle, concomitantly with a reduction in the melting enthalpy. A similar effect has been identified by other authors in studies on mixes of PLA with epoxy functionalized plasticizers [13] and the difficulty of predicting the likely disappearance of the double melting peak in blends has also been reported in the literature. For instance, in the case of the work by Zhang et al. the double peak was found only on annealed samples [16], while Jaratrotkamjion et al. have observed that the splitting of the single peak does not take place in the second heating scan [18]. It should be noted that the very small increase in $\mathrm{T}_{\mathrm{g}}$ of ENR50 after exposures to high temperatures has 
been observed before and it has been attributed to the opening of a small number of oxirane rings along the chains through reaction with water, rather than cross-linking $[18,24]$.

\subsection{Observations of events during cooling from the melt and heating from the glassy state.}

The thermograms recorded for the DSC runs at different cooling rates are shown in Fig.

3. For the sake of clarity, in all cases the curves are displaced downwards in order of increasing rate of temperature change used in the measurements, distinguishable also by the colour of the traces.

a)

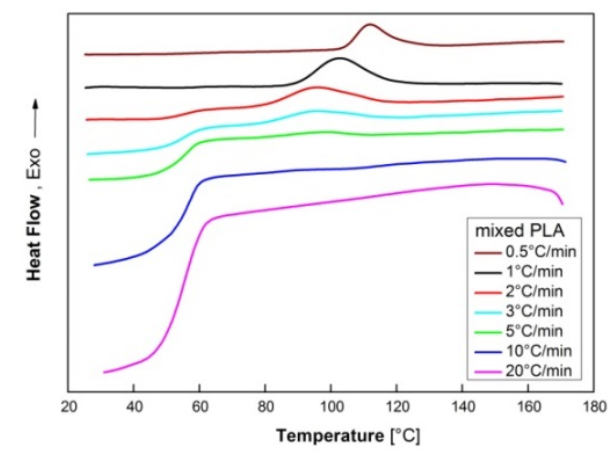

c)

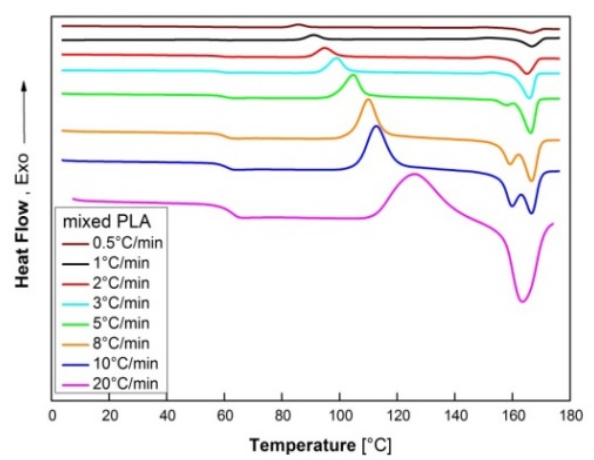

b)

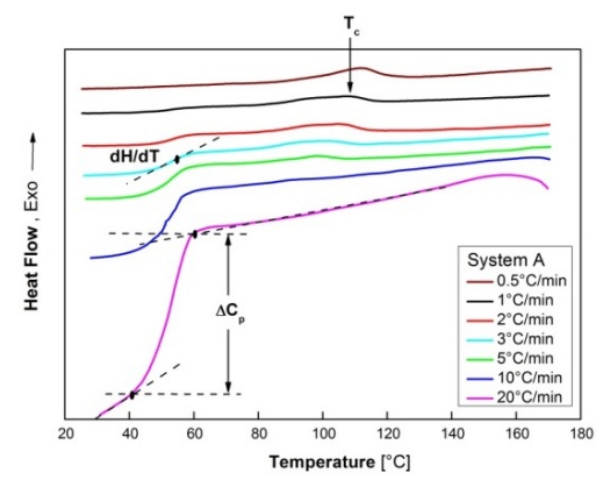

d)

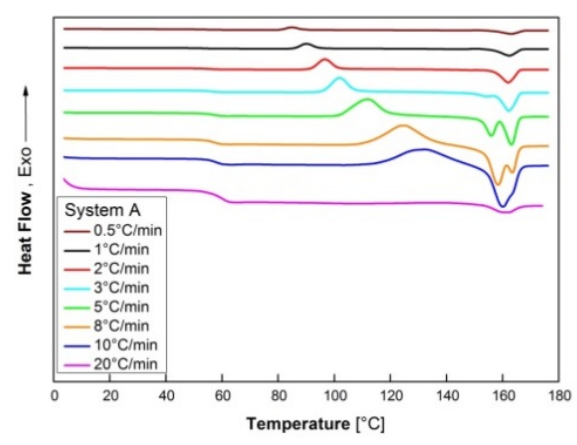

Fig. 3. DSC Thermograms at different rate of temperature change for systems based on crystalline PLA. Top curves are traces from cooling experiments a) mixed PLA, b) System A.

Bottom curves are the related traces for heating experiments: c) mixed PLA, d) System A.

Visual observations on the thermograms in Fig. 3 reveal some significant differences for the effect of the direction of the imposed temperature change in relation to the crystallization behaviour of both samples: most notable is the very large suppression of the crystallization peaks and the much steeper calorimetric traces at the high cooling rates. A splitting of the melting peaks is displayed by thermograms for both systems at the higher heating rates, starting from $3{ }^{\circ} \mathrm{C} / \mathrm{min}$, while a convergence of two contiguous crystallization 
peaks for System A is observed at cooling rates from about 1 to $3{ }^{\circ} \mathrm{C}$ (Fig. $3 \mathrm{~b}$ ). It should be noted that a crystallization double peak for PLA has been observed during the heating cycle in experiments carried out by Liao et al. [25] but no similar observations have been reported for crystallization from the melt state. In studies of the crystallization behaviour of PLA solvent-cast films Byun et al. [26] have found that the formation of a single or double melting peak depends on the nature of the solvent, which determine whether mostly the PLA $\alpha$-phase is formed or whether a mixture of $\alpha, \beta$ and $\gamma$ crystals.

Another observation regarding the thermograms in the heating mode is the merging of the crystallization and melting peaks at high heating rates, as also reported by Pyda et al. [27]. For experiments at $20{ }^{\circ} \mathrm{C} / \mathrm{min}$ the convergence of the two events brings about an almost complete obliteration of the melting endotherm for melt-quenched System $\mathrm{A}$ and a broadening of the exotherm for mixed PLA, concomitantly with the loss of the double melting peak displayed at lower heating rates. This is yet another example of the sporadic melting behaviour of PLA rooted in the thermal history of the sample and a confirmation of the effect of the temperature scanning rate used in the measurements.

The enthalpy data for the thermograms in Fig. 4 reveal contrasting trends with respect to the direction of the temperature scan and inverse effects of the rubber modification on the crystallization behaviour. The reduction in crystallization enthalpy with increasing cooling rate (Fig. 4a), leading to a total suppression at some critical value, is attributed to the rate of reduction in temperature relative to the rate of depression of molecular relaxation rate involved in the formation of crystals. At the same time, the lower normalized $\Delta H c$ values for System A indicate that the rubber modification hinders the crystallization during cooling by exerting greater constrictions to molecular relaxations than for PLA in isolation.

Regarding the data on crystallization by heating from the pristine glassy state, on the other hand, the plots in Fig. $4 \mathrm{~b}$ show that the $\Delta H c$ values increase with cooling rate up to a maximum, which results from the overlapping of crystallization and melting events. In measurements at $20{ }^{\circ} \mathrm{C} / \mathrm{min}$ heating rate for System A the total suppression of crystallization coincides with the extremely small extent of melting of the crystalline species. The recorded values are $3.0 \mathrm{~J} / \mathrm{g}$ for $\Delta \mathrm{Hm}$ and $0.0 \mathrm{~J} / \mathrm{g}$ for $\Delta H c$ for System A, while the corresponding values for the mixed PLA are respectively $31.2 \mathrm{~J} / \mathrm{g}$ and $26.9 \mathrm{~J} / \mathrm{g}$. 
a)

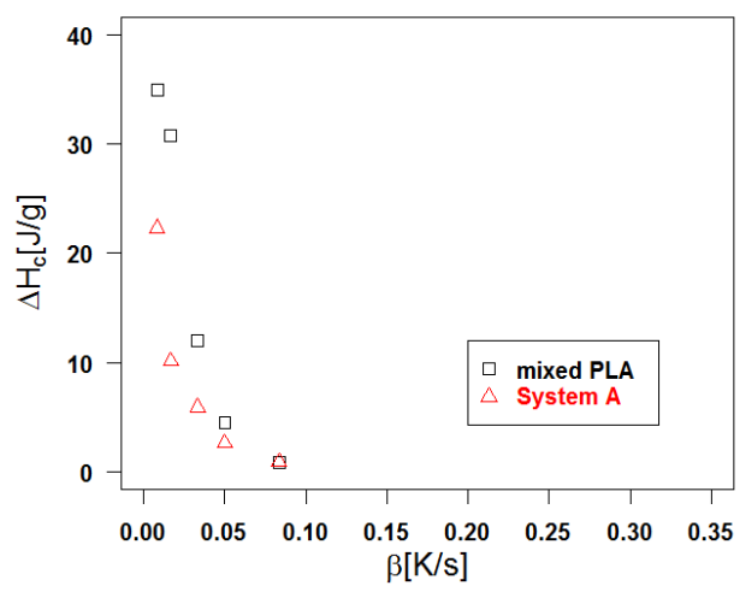

b)

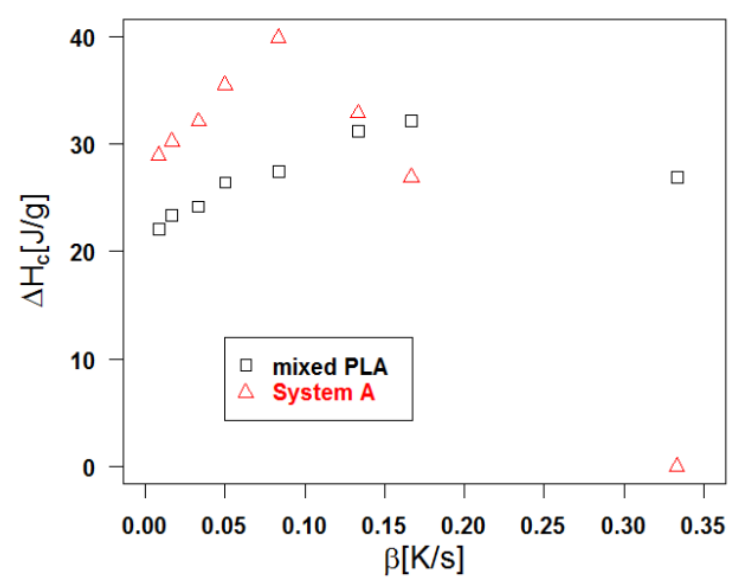

Fig. 4. a) $\Delta H_{c}$ versus $\beta$ for cooling experiments from the melt state, b) $\Delta H_{c}$ versus $\beta$ for heating experiments on melt quenched samples from the "pristine" glassy state.

Nevertheless, the higher $\Delta H c$ values for System A for heating rate conditions below those corresponding to the maximum for the heat of crystallization indicate that the presence of solubilized ENR50 species in the PLA assists the crystallization of the sample when heated from the pristine glassy state by increasing the rate of molecular relaxation required for the growth of crystals, through a solvation effect.

\subsection{Crystallization kinetics}

The kinetics of crystallization under non-isothermal conditions are often examined using the Kissinger method through the equation below [28, 29]

$$
\ln \left(\beta / T_{c}{ }^{2}\right)=\ln (-A R / E) f(\alpha)-E / R T_{c}
$$

where $\beta$ = heating or cooling rate, $A=$ pre-exponential constant in the Arrhenius equation, $E=$ activation energy and $T_{C}=$ the peak crystallization temperature.

If the activation energy is constant for the entire crystallization process its value can be obtained directly from plots of $\ln \left(\beta / T_{c}{ }^{2}\right)$ versus $1 / T_{c}$. The plots for the materials of this study are shown in Fig. 5. 
To test the reproducibility of the data and the efficacy of the memory erasing treatment of the two materials cooled from the melt state the experiments were repeated on samples that were previously annealed for 20 minutes at $120{ }^{\circ} \mathrm{C}$ in order to increase the inherent level of crystallinity.

a)

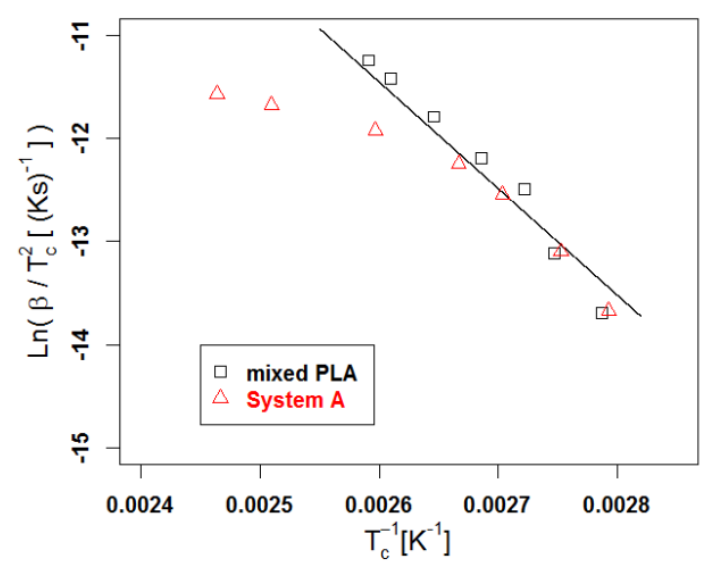

b)

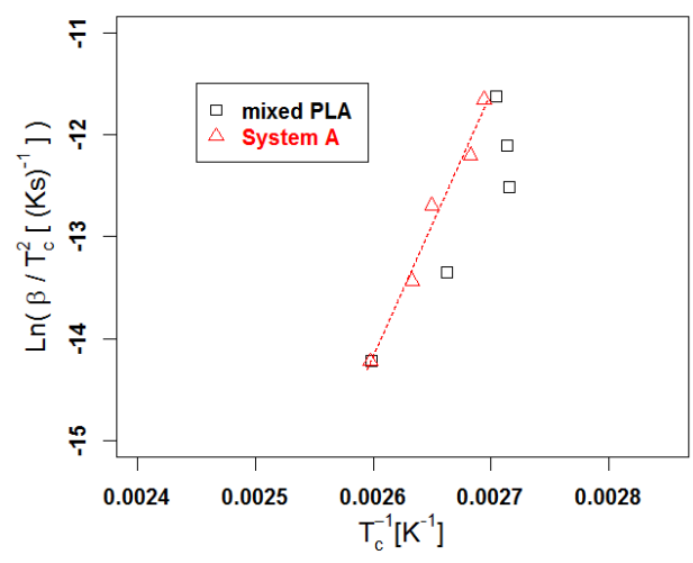

Fig. 5. (a) Kissinger plots for the crystallization of mixed PLA and System A by heating from the glassy state. (b) Fictitious plot for the same materials using DSC data obtained for crystallization from the melt state.

For experiments carried out in the heating mode from the pristine glassy state a reasonably good fit is obtained for the Kissinger equation on samples of the mixed, due to appearance of distinct crystallization peaks in the related thermograms. The loss of linearity in the plots for System A at high heating rates, on the other hand, can be attributed to the merging of the crystallization and melting events, which affects the recorded values for both $T c$ and $\Delta H c$ as evidenced by the downward slope of the calorimetric trace before the start of the melting peak at heating rates above $5{ }^{\circ} \mathrm{C} / \mathrm{min}$. At low heating rates the two systems exhibit similar activation energy. Irrespective of these anomalies, however, the results reveal clearly the significant difference brought about by the change in direction of the temperature scan, and the inversion of the effects of the interactions between the PLA phase and the rubbery component on the activation energy for the crystallization events, in concordance with the $\Delta H c$ plots shown in Fig. 4. 
Fictitious Kissinger plots for the crystallization experiments carried out by cooling the samples from the melt state are shown in Figure 5 (b) as a graphical comparison with those derived for data obtained in the heating mode. One notes a change in the direction of the gradient of the plots from the graphs derived for the experiments in the heating mode, indicating that the activation energy is negative. A communication by Vyazovkin [30] questions the validity of the practice of using Kissinger plots to calculate the activation energy for crystallization induced by cooling from the melt by arguing that changing the direction of the temperature ramp in DSC experiments requires a change in the sign of $\beta$ in equation 1 . This brings about a concomitant change in the signs of the parameters in the right side of the equation, which induces the activation energy to assume a negative value and, therefore, produces a paradox with respect to its physical meaning. Although one could argue that this is an artificial problem that can be solved by inverting the direction of $\beta$ along the abscissa, Vyazovkin reminds that the Kissinger equation derives from the general rate equation

$$
\frac{d \alpha}{d t}=A \exp \left(\frac{-E}{R T}\right) f(\alpha)
$$

which predicts a maximum in the conversion rate as a function of $\beta$ (i.e. $d T / d t$ ), which is obtained by setting the derivative equal to zero, where the value of $T$ corresponds to the peak temperature $T c$ in equation 1.

Introducing a negative sign in the parameter $\beta$, in concordance with the direction of the change in temperature in cooling experiments, and setting to zero the derivative the solution of the equation produces a minimum. This is at variance with the data recorded in the related thermograms, which show a maximum in the heat of crystallization irrespective of the direction of $\beta$. Using data obtained on the crystallization of PET from the melt state [31] To Vyazovkin overcomes this dilemma by introducing a modification of the Kissinger method to obtain values for the "effective" activation energy, which would replace the fictitious value obtained from the conventional plots, as in Figure 5 (b), For the material used by Vyazovkin as an example not only the peak crystallization temperature increases monotonically with increasing the cooling rate but the actual exothermal peaks are distinct and clearly identified. This is not the case for the thermograms recorded in the cooling experiments on the PLA based materials used in the present study: The blend System A shows a maximum in the variation of Tc with cooling rate (see Figure 3a) and the thermograms for the mixed PLA sample exhibit very shallow broad exotherms and ill-defined double peaks (Figure $3 \mathrm{~b}$ ). 
These features, therefore, constitute a substantial hindrance to any tentative effort to obtain a more elaborate analysis of the crystallization kinetics for the materials of this study in relation to experiments carried out in the cooling mode.

\subsection{Vitrification kinetics}

In addressing the variation of vitrification enthalpy with heating rate, Hodge has used the concept of a "characteristic relaxation time" $(\tau)$ [32], while Aou et al. [33] have used the Arrhenius equation to calculate the activation energy by replacing the relaxation time with cooling rate for studies on different types of amorphous PLA. The latter authors have recorded the values of the temperature for the onset of the glass transition $\left(T_{g(i)}\right)$, which were then used to produce plots of $\ln \beta$ versus $1 / T_{g(i)}$. This is an adaptation of Friedman isoconversional method [34] for non-isothermal runs, which relates the degree of conversion to form a glass $(\alpha)$ to the change in enthalpy, i.e. $\frac{d \alpha}{d t}=k \frac{d H}{d t}$, where $k$ is a proportionality constant.

Therefore, a relationship for events during vitrification and de-vitrification can be written as

$$
k \frac{d H}{d T}=\frac{d \alpha}{d t} \frac{1}{\beta}
$$

where $\beta=d T / d t$ and the term $\frac{d \alpha}{d t}$ can be related to temperature $(T)$ through the Arrhenius equation, i.e. $\frac{d \alpha}{d t}=A e^{-E / R T}$.

Hence

$$
\frac{d H}{d T}=A^{*} \frac{e^{-E / R T}}{\beta}
$$

where $A^{*}$ is a modified pre-exponential constant.

The fitting of the DSC data by Aou et al. to the above equation for the vitrification of a glassy polymer is rooted in the premise that the effect of temperature on the rate of change in molecular relaxations within the glass transition region is equivalent to the change in the glass transition temperature with respect to changes in the cooling rate. This leads to the expression 


$$
\ln \left(\frac{d H}{d T} \frac{\beta}{A *}\right)=-\frac{E}{R T g}
$$

For constant $d H / d T$ within the glass transition region, plots of $\ln \beta$ versus $1 / T g$ (known also as Mahadevan plots [35]) should produce a straight line with gradient equal to E/R.

The validity of Hodge's premise hinges on the relationship between the change in enthalpy for vitrification under isothermal conditions to the relaxation time of the system $(\tau)$, which can be described by the equation $H(t)=\left(H_{o}-H_{\infty}\right) \exp [-(t / \tau)]$. It should be possible, therefore, to use a similar approach for the temperature derivative $d H / d T$ expressed as a function of the parameter $(\dot{\vartheta})$, which corresponds to the temperature derivative of the relaxation time and represents the structural factor controlling the change in enthalpy within the glass transition region, i.e. $d H / d T=f(\beta / \dot{\vartheta})$ for non-isothermal conditions, and is expected to follow a similar exponential law. This would allow the factor $\dot{\vartheta}$ to be related to temperature via the Arrhenius equation as indicated in equation 5.

A more practical approach can be used to analyse the DSC data for vitrification and de-vitrification studies from non-isothermal measurements at different rates of temperature changes, simply through direct observations on the changes in $\Delta C_{p}$ recorded for the glass transition as plots against $\beta$, shown in Fig. 6.

a)

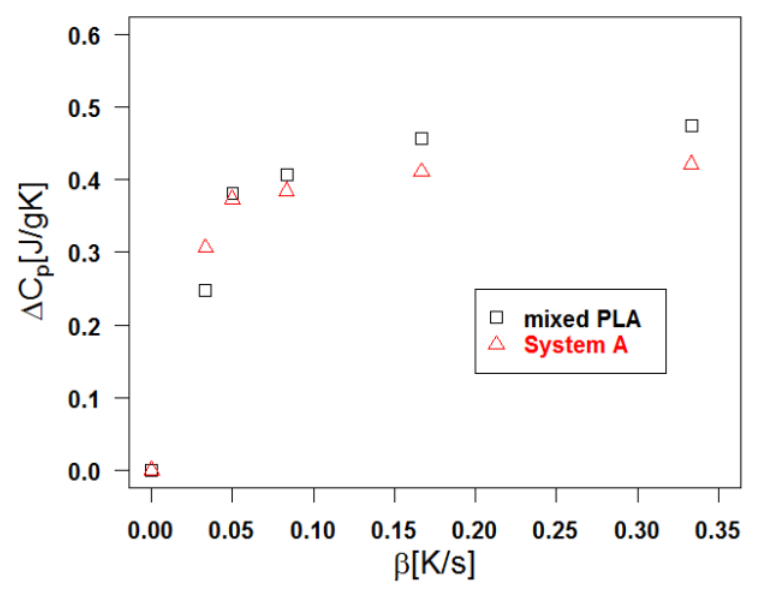

b)

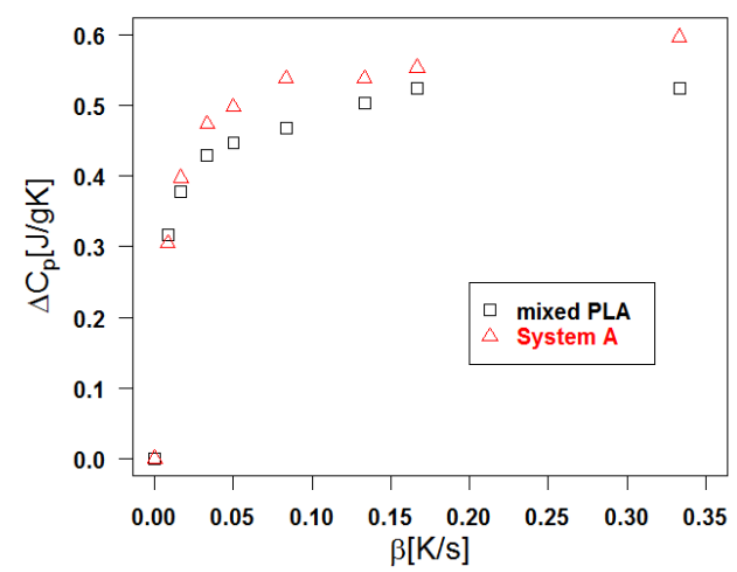

Fig. 6. Plots of $\Delta C_{p}$ against temperature variation rate $(\beta)$ for mixed PLA and System A systems obtained from DSC thermograms in Fig. 2.

a) Cooling experiments from the memory-free melt state. b) Heating experiments from melt-quenched samples (pristine glassy state). 
The changes in $\Delta C_{p}$ with increasing temperature variation rate $(\beta)$ are related to the suppression of molecular relaxations by an auto-retardation mechanism for measurements in the cooling mode and to the auto-acceleration of the molecular relaxations for de-vitrification while approaching the rubbery state. Accordingly, the higher $\triangle C p$ values resulting from the presence of the rubbery component of the blend in the de-vitrification runs are associated with the lower density of the blend due to plasticization effects, while the corresponding higher $\Delta H_{c}$ values are a consequence of the solvent-induced acceleration of the subsequent crystallization events. On the other hand, the somewhat lower $\Delta C_{p}$ values identified for the vitrification at high cooling rates, shown in Fig. 6, can be taken as a memory effect of the crystallinity in the form of a higher level of molecular order in the resulting glass (see effects on $\mathrm{T}_{\mathrm{g}}$ in the following discussion).

The variations of the recorded values of $T_{g}$ with the rate of temperature change for the materials examined is shown in Fig. 7.

a)

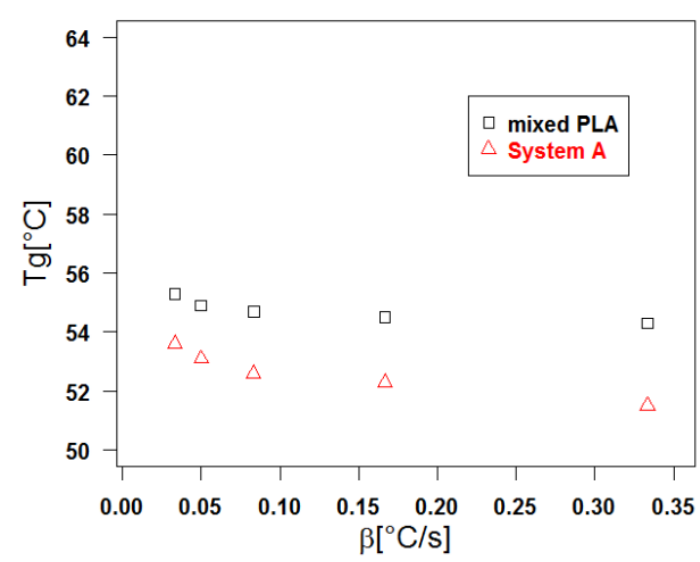

b)

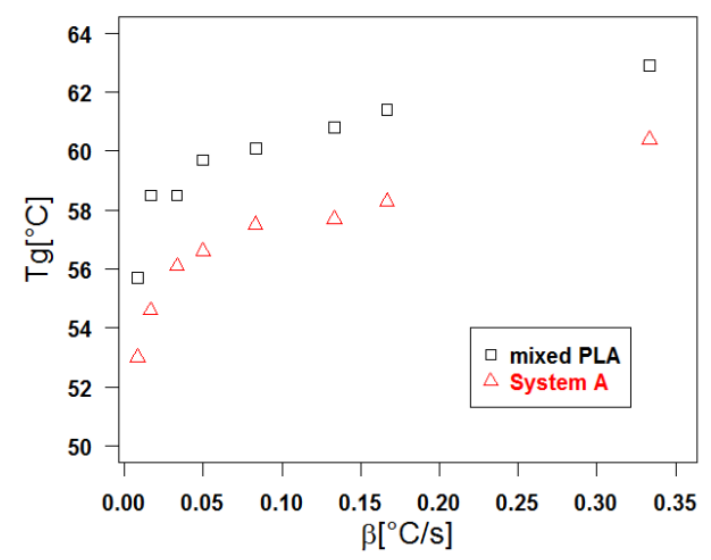

Fig. 7. Variation of $T_{g}$ with temperature variation rate for mixed PLA and System A.

a) Data for DSC cooling runs from the memory-erased melt state.

b) Data for DSC heating runs from the melt-quenched (pristine) glassy state.

For cooling experiments carried out from the memory-erased melt state the plots display a decrease in $\mathrm{Tg}$ with increasing cooling rate. This is a similar trend to that observed for the heat of crystallization in Fig. $4 a$. The higher $\mathrm{Tg}$ values at lower cooling rates are attributable to the higher molecular order established within the amorphous regions as a 
"hindrance" effect on molecular relaxations by the crystalline domains, which persist as a pseudo-order even at high cooling rate when the recorded $\Delta H_{c}$ values are equal to zero. An opposite trend is obtained, on the other hand, for experiments carried out in the heating mode from a pristine glassy state (Fig. 7 b). While both experimental conditions show the expected reduction in $\mathrm{Tg}$ for System A, due to plasticization effects of the rubbery component, the heating mode brings about a large increase in the recorded $\mathrm{Tg}$ values with temperature scanning rate. Furthermore, for experiments in the heating mode, the recorded $T g$ are much higher than for the cooling mode, which is indicative of a delay in the relaxations for the devitrification into a rubber.

The Mahadevan plots using the values of $\mathrm{Tg}$, in accordance with equation 4, rather than $T g_{(i)}$ used by Aou et al [33], for the two experimental conditions, are shown in Fig. 8.

a)

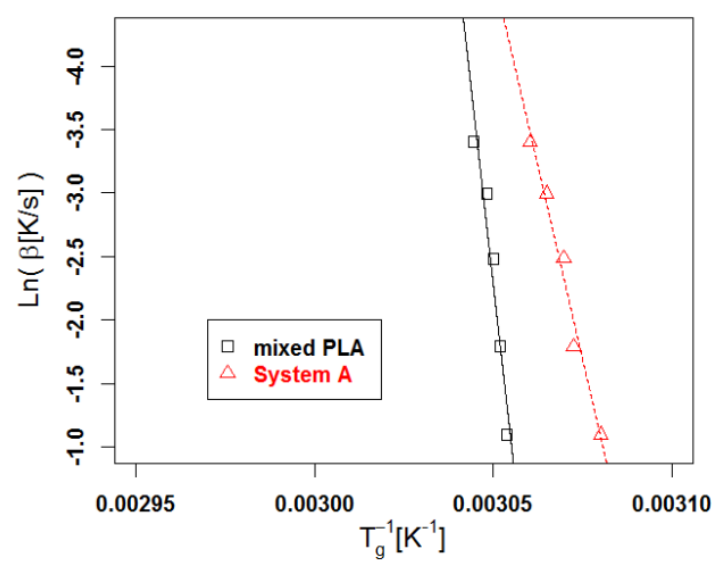

b)

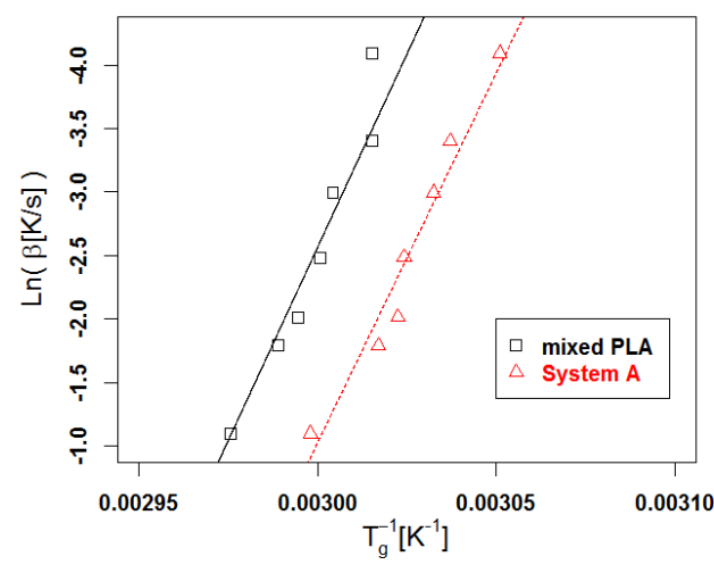

Fig. 8. Mahadevan plots for modeling the variation of $T_{g}$ with changes in the temperature ramp $\beta$ for mixed PLA and System A.

a) Data for DSC cooling runs from the melt state. b) Data for DSC heating runs from the (pristine) glassy state.

For the heating scans the values are respectively $0.50 \mathrm{MJ} / \mathrm{mol}$ for mixed PLA and 0.48 $\mathrm{MJ} / \mathrm{mol}$ for System A. These values are in the same order of magnitude of ones reported by Aou et al. [32] for a variety of grades of PLA, which were found to fall within the range 750 - $900 \mathrm{~kJ} / \mathrm{mol}$. The numerical difference for the obtained values is probably related to the difference in grades of PLA and also to the use of $\mathrm{Tg}$ in this work instead of $\mathrm{Tg}(\mathrm{i})$. The very high numerical values obtained in both works are a reflection of the relatively high sensitivity 
of $\mathrm{Tg}$ to the rate of change in temperature within the possible range achievable for the glass transition region.

The plots in Figure 8 again exhibit a reversion of the direction of the gradient produced from data obtained in cooling experiments. Although it is legitimate to calculate the activation energy of the devitrification events in heating from the glassy state, questions can again be raised about the validity of using the same procedure for vitrification by cooling from the melt state. It is noted that in the Mahadevan plots for the amorphous PLA samples the gradient is positive for both heating and cooling measurements. This behavior results from the relationship between $\mathrm{dH} / \mathrm{dt}$ and $\beta$, whereby changing the sign of one parameter brings about a concomitant change in the other. Equation 5 shows that the product of these two terms is always positive and, therefore, the gradient of the derived plot remains unaltered. The reason for the negative gradient in the plots produced from cooling experiments for the two materials based on crystalline PLA is rooted in the difference in the variation of Tg with $\beta$, as shown in Figure 7, which was discussed earlier. Accordingly, the vitrification of samples based on crystalline PLA could be regarded as a dual process in which the molecular dynamics would still tend towards the formation of ordered domains, even when the preceding crystallization step is prevented by the imposition of a rapid cooling rate. This suggests, therefore, that the vitrification kinetics may have to be considered along similar lines taken by Vyazovkin for crystallization induced by cooling from the melt state. Consequently no attempt was made to calculate the activation energy for these systems.

\subsection{Comparison with intrinsically amorphous PLA}

To verify that the differences identified regarding the effects of the heat flow direction is peculiar to crystalline PLA, similar experiments and analysis were performed on an amorphous grade of PLA that had received a similar thermal processing treatment. The plots in Fig. 9a show that the $\mathrm{Tg}$ increases with increasing scanning temperature rate irrespective of the heat flow direction used in the DSC measurements. 

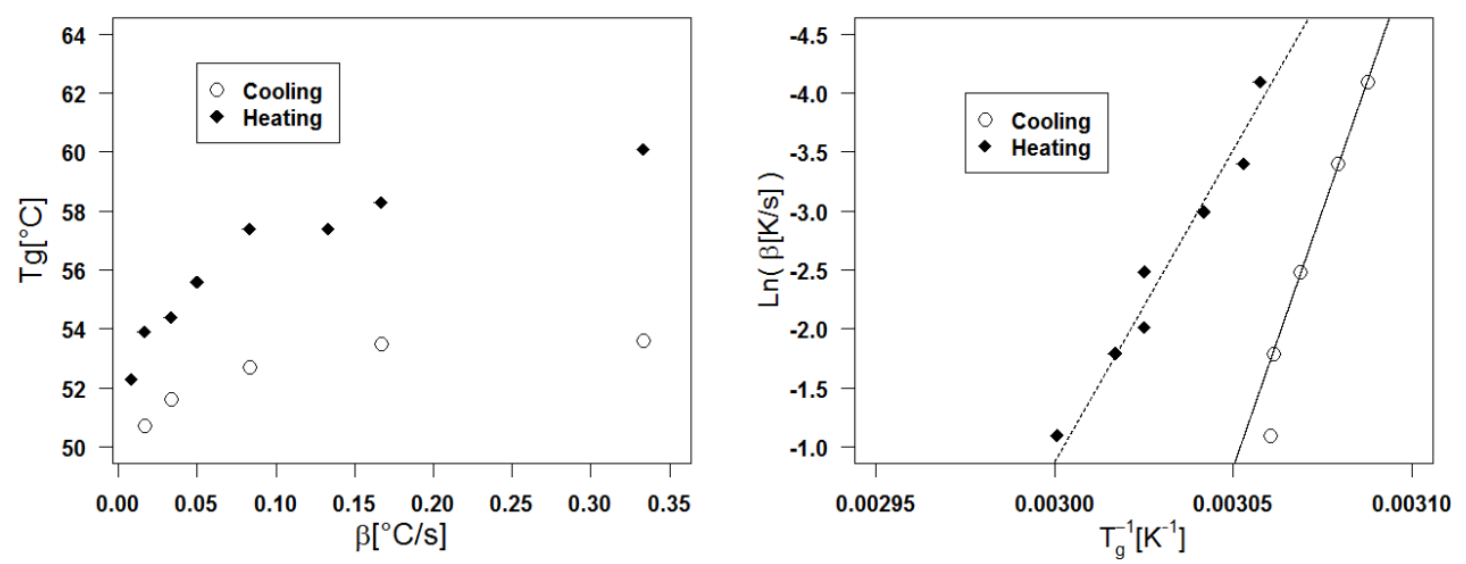

Fig. 9. Vitrification thermal parameters for amorphous PLA.

a) Variation of $T_{g}$ with rate of temperature variation $\beta$ ) for runs carried out by DSC in cooling and heating modes. b) Mahadevan plots for modeling of $T_{g}$ with rate temperature variation $(\beta)$ for the two temperature scanning modes.

From this observation and from the foregoing discussion it is evident that the chain regularity in the crystallizable grade of PLA affects the behavior of the derived materials with respect to the molecular relaxations involved in events within the glass transition region. When cooling the crystallizable PLA from a memory free melt state, the vitrification transition takes place after crystallization and, therefore, it induces a global higher degree of order alongside a greater restriction to the mobility of the chains during subsequent vitrification, which lead to somewhat higher $T g$ values in comparison to those obtained for the amorphous grade under the same experimental conditions. The results indicate also that chain regularity allows the material to acquire a degree of order in the glassy region even at higher cooling rate when crystallization is suppressed by the unfavourable conditions for the formation of crystals. Reducing the chain regularity in the synthesis of an intrinsically amorphous PLA grade increases the propensity of the polymer chains to assume random molecular configurations, which prevent the establishment of the same degree of order in the glassy state as that achievable with crystallizable grades. This allows a broader range of spatial configurations, leading to a wider range of free volumes achievable through variations in thermal history conditions and, therefore, also to a wider range of $\mathrm{Tg}$ values recorded in DSC scans. The crystalline grade of the examined PLA displays higher $T g$ values also for measurements in the heating mode and, therefore, this behavior has to be related to intrinsic differences in molecular structure.

The estimated activation energy values for the amorphous PLA grade obtained in the two runs are $723.7 \mathrm{~kJ} /$ mole in the cooling mode and $439.8 \mathrm{~kJ} / \mathrm{mol}$ in the heating mode. The 
latter value is similar to that reported earlier for the crystalline PLA (480 kJ/mol), which occurs in concordance with the similarity displayed with respect to the upper $T g$ values at high heating rates. The equivalence of the data obtained in the heating mode for the amorphous and crystalline PLA based systems, therefore, must be taken as a reflection of the lack of molecular restrictions in the absence of crystalline domains in the pristine glassy state.

\section{Conclusions}

A crystalline grade of PLA was mixed with $10 \mathrm{w} \%$ of ENR50, alongside control runs consisting of both crystalline and amorphous PLA, and the thermochemical characteristics were evaluated by monitoring the change viscosity during mixing and by performing a routine DSC analysis and TGA run. The blend was found to display some peculiarities with respect to thermal transitions and to be more thermally unstable than the individual component.

The kinetics for crystallization and glass-rubber transitions were studied by performing non-isothermal DSC runs in both heating and cooling modes. The DSC data have identified a relatively small plasticization effect due to presence of solubilised ENR species in the PLA domains, resulting from transesterification reactions between the two components.

It was also found that the classical Kissinger crystallization kinetics analysis for experiments carried out in the heating mode is applicable only to the unmodified PLA system. The reactive blend displayed a monotonic increase in activation energy with increasing heating rate.

The vitrification kinetics, on the other hand, could be accurately modelled for all systems using the Mahadevan method. While very little difference could be detected between the crystalline and amorphous grades of PLA for devitrification monitored by heating the sample from a pristine glassy state, the crystallization events taking place prior to the onset of vitrification when cooling from the melt state were found to exert a pronounced effect not only on the activation energy but also with respect to the variation of $\mathrm{Tg}$ with changes in cooling rate.

The presence of solubilized species from the rubbery component of the blend was found to have contrasting effects on the heat of crystallization of PLA, displaying an attenuation of the $\Delta H c$ values with cooling rate for vitrification from the melt and an 
enhancement in the heat of crystallization (degree of crystallinity) when heating from a pristine glassy state.

\section{References}

[1] L. Mascia, Polymer Compositions, European Patent No. 84305078.2, (1985).

[2] X. Zhang, Y. Li, L. Han, C. Han, K. Xu, C. Zhou, M. Zhang, L. Dong, Improvement in toughness and crystallization of poly(L-lactic acid) by melt blending with ethylene/methyl acrylate/glycidyl methacrylate terpolymer, Polym. Eng. Sci. 53 (2013) 2498-2508.

[3] N. Petchwattana, S. Covavisaruch, and N. Euapanthasate, Utilization of ultrafine acrylate rubber particles as a toughening agent for poly(lactic acid), Mater. Sci. Eng. A 532 (2012) 64-70.

[4] A.M. Gajria, V. Dave, R. A. Gross, and S. P. McCarthy, Miscibility and biodegradation of blends of poly (lactic acid) and poly (vinyl acetate), Polymer 37 (1996) 437-444.

[5] H.T. Oyama, Super-tough poly(lactic acid) materials: Reactive blending with ethylene copolymer, Polymer 50 (2009) 747-775.

[6] J. W. Park and S. S. Im, Miscibility and morphology in blends of poly (L-lactic acid) and poly(vinyl acetate-co-vinyl alcohol), Polymer 44 (2003) 4341-4354.

[7] Y.F. Kim, C. N. Choi, Y.D. Kim, K.Y. Lee, M.S. Lee, Compatibilization of immiscible poly(l-lactide) and low density polyethylene blends, Fibers and Polymers 5 (2004) 270-274.

[8] K. S. Anderson and M. A. Hillmyer, The influence of block copolymer microstructure on the toughness of compatibilized polylactide/polyethylene blends, Polymer 45 (2004) 8809-8823.

[9] G. Biresaw and C. J. Carriere, Interfacial tension of poly(lactic acid)/polystyrene blends, Journal of Polymer Science, Part B: Polymer Physics 40 (2002) 2248-2258.

[10] H. Liu, J. Zhang, Research Progress in Toughening Modification of Poly(lactic acid), J. Polym Sci Part B: Polym Phys 49 (2011) 1051-108.

[11] B. Imre and B. Pukánszky, Compatibilization in bio-based and biodegradable polymer blends, Eur. Polym. J. 49 (2013) 1215-1233. 
[12] V. Vilay, M. Mariatti, Z. Ahmad, K. Pasomsouk, M.Todo, Characterization of the mechanical and thermal properties and morphological behaviour of biodegradable poly(L-lactide)/poly( $\varepsilon$-caprolactone) and poly(L-lactide)/poly(butylene succinate-coL-lactate) polymeric blends, J. Appl. Polym. Sci. 114 (2009) 1784-1792.

[13] L. Wang, W. Ma, R.A. Gross, S.P. McCarthy, Reactive compatibilization of biodegradable blends of poly(lactic) acid and poly(caprolactone), Polym. Degrad. Stabil. 59 (1998) 161-168.

[14] X. Cao, a. Mohamed, S. H. Gordon, J. L. Willett, and D. J. Sessa, DSC study of biodegradable poly(lactic acid) and poly(hydroxy ester ether) blends, Thermochim. Acta 406 (2003) 115-127.

[15] C. Zhang, W. Wang, Y. Huang, L. Jiang, Y. Dan, Y. Luo, Z. Peng, Thermal, mechanical and rheological properties of polylactide toughened by epoxidized natural rubber, Materials and Design 45 (2013) 198-205.

[16] J. K. Mishra', Y. W. Chang, D.K. Kim, Green thermoplastic elastomer based on polycaprolactone/epoxidized natural rubber blend as a heat shrinkable material, Materials Letters, 61 (2007) 3551-3554.

[17] R. Jaratrotkamjom, C. Khaokong, V. Tanrattanakul, Tougheness enhancement of poly(lactic acid) my melt blending with natural rubber, J. Appl. Polym. Sci. 124 (2012) $5027-5036$.

[18] L. Mascia, P. Russo, L. Verdolotti, J. Clarke, M. Lavorgna, D. Acierno, Probing the post-gelation reactions of epoxidized natural rubber cross-linked with dodecenyl succinic anhydride. Rubber Chemistry and Technology, 88 (2015) 560 - 573.

[19] H. Li, M. A. Huneault, Effects of nucleation and plasticization on the crystallization of poly(lactic acid), Polymer 48 (2007) 6855-6866.

[20] Z. Kulinski, E. Piorkowska, Crystallization, stricture and properties of plasticized poly(L-lactide), Polymer 46 (2005) 10290 - 10300.

[21] D. Garlotta, A literature review of poly(lactic acid), Journal of Polymers and the Environment, 9 (2001) $63-84$.

[22] C. Liu, S. Lin, C. Zhou, W. Yu, Influence of catalyst on transesterification between poly(lactic acid) and polycarbonate under flow field, Polymer, 54 (2012) 310-319.

[23] M. Nofar, A. Ameli, C.B. Park, Development of polylactide bead foams with double crystal melting peaks, Polymer 69 (2015) 83 - 94.

[24] L. Mascia, J. Clarke, K.- S. Ng, K. S. Chua, P. Russo, Curing efficiency of Dodecyl Succinic Anhydride as a Cross-Linking Agent for Elastomer Blends Based on 
Epoxidized Natural Rubber, J. Appl. Polym. Sci., 132 (2015) 1; DOI:101002/app 41448

[25] K. Liao, D. Quan, Z. Lu, Effects of physical aging on glass transition behaviour of poly(DL-lactide), European Polymer J. 38 (2012) 157 - 162.

[26] Y. Byun, S. Whiteside, R. Thomas, M. Dhaman, J. Hughes, Y.T. Khim, The effect of solvent mixtures on the properties of solvent cast films, J. Appl. Polym. Sci, 124 (2012) 3577- 3582].

[27] M. Pyda, R.C. Bopp, B. Wunderlich, Heat capacity of poly(lactic acid), J. Chem. Thermodynamics 36 (2004) 731-742

[28] H.F. Kissinger, Reaction kinetics in differential thermal analysis, Analytical Chemistry 29 (1957) 1702-1706.

[29] S. Vyazovkin, A.K. Burnham. J.M. Criado, L.A., Perez-Maqueda, ICTAC Kinetics Committee recommendations for performing kinetic computations on thermal analysis data, Thermochimica Acta 520 (2011) 1-19.

[30] S. Vyazovkin, Is the Kissinger equation applicable to the processes that occur on cooling?, Macromol. Rapid Commun. 23 (2002) 771-775.

[31] S. Vyazovkin and N. Sbirrazzuoli, Isoconversional analysis of the non-isothermal crystallization of a polymer melt, Macromol. Rapid Commun. 23 (2002) 766 -770.

[32] Hodge I. M., Enthalpy relaxation and recovery of amorphous materials, J. Non_Cryst. Solids 169 (1994) 211-264.

[33] K. Aou, S.L. Hsu, L.W. Kleiner, F_W. Tang, Roles of conformational and configurational defects on the physical aging of amorphous poly(lactic acid), J. Phys. Chem. 111 (2007) 12322-12327.

[34] H.L. Friedman. Kinetics of thermal degradation of char-forming plastics from thermogravimetry: Application to a phenolic plastic, J. Polym. Sci. Part C 6 (1964) $183-195$.

[35] S. Mahadevan, A. Giridhar, A.K. Singh, Calorimetric measurements on As-Sb-Se glasses, J. Non-Cryst. Solids, 88 (1986) 11-34. 